\title{
Lung Texture Classification Using Locally-Oriented Riesz Components
}

\author{
Adrien Depeursinge ${ }^{1,2}$, Antonio Foncubierta-Rodriguez ${ }^{1}$, \\ Dimitri Van de Ville ${ }^{2,3}$, and Henning Müller ${ }^{1,2}$ \\ 1 University of Applied Sciences Western Switzerland (HES-SO) \\ 2 University and University Hospitals of Geneva (HUG), Switzerland \\ 3 Ecole Polytechnique Fédérale de Lausanne (EPFL), Switzerland \\ adrien.depeursinge@hevs.ch
}

\begin{abstract}
We develop a texture analysis framework to assist radiologists in interpreting high-resolution computed tomography (HRCT) images of the lungs of patients affected with interstitial lung diseases (ILD). Novel texture descriptors based on the Riesz transform are proposed to analyze lung texture without any assumption on prevailing scales and orientations. A global classification accuracy of $78.3 \%$ among five lung tissue types is achieved using locally-oriented Riesz components. Comparative performance analysis with features derived from optimized grey-level co-occurrence matrices showed an absolute gain of $6.1 \%$ in classification accuracy. The adaptability of the Riesz features is demonstrated by reconstructing templates according to the first principal components of the lung textures. The balanced performance achieved among the various lung textures suggest that the proposed methods can complement human observers in HRCT interpretation, and opens interesting perspectives for future research.
\end{abstract}

Keywords: Texture analysis, Riesz transform, interstitial lung diseases, high-resolution computed tomography, computer-aided diagnosis.

\section{Introduction}

Successful diagnostic interpretation of medical images relies on two distinct processes. First, abnormal image patterns are identified (e.g., fibrous tissue, architectural distortion, ...) and, second, links between the patterns and possible diagnoses can be established [1. Whereas the latter requires a deep understanding and comprehensive experience of the involved diseases, the former is closely related to visual perception. Interestingly, a large scale study on malpractice in radiology showed that the majority of errors in medical image interpretation are caused by perceptual misapprehensions 2. Texture analysis is central to human image understanding and plays an important role in efficient characterization of biomedical tissue, that cannot be described in terms of shape or morphology [3. As a consequence, computer-aided diagnosis (CAD) based on texture quantification in radiological images has been an active research field over the

G. Fichtinger, A. Martel, and T. Peters (Eds.): MICCAI 2011, Part III, LNCS 6893, pp. 231-238, 2011. (C) Springer-Verlag Berlin Heidelberg 2011 
past 20 years with the aim of reducing omission errors of pathological tissue by providing systematic second opinions to radiologists.

Texture analysis is the cornerstone for differentiating between pathological and healthy lung tissue of patients affected by interstitial lung diseases (ILD) in high-resolution computed tomography (HRCT). ILDs group more than 150 disorders of the lung tissue of varying origin and can be differentiated only by detecting subtle texture changes of the lung parenchyma with a characteristic distribution within the lung anatomy [4]. Interpreting HRCT images of the chest represents a challenge even for trained radiologists and lung specialists. Several studies have been conducted on the use of computerized lung texture classification to assist the radiologists in HRCT interpretation for ILDs or chronic obstructive pulmonary disease (COPD) starting from 1997 [56/7/8]. The success of the CAD system is intimately related to the ability of the visual features to catch and learn the subtle texture signatures specific to each lung tissue type, which are typically non-deterministic. Therefore, statistical approaches that are able to capture texture properties at any location, scale and orientation (i.e., affine-covariant) are required to achieve high tissue classification performance to complement human observers. Whereas more than 60 papers using texture analysis to classify lung tissue can be found in the literature of the past 15 years [9], research contributions on novel texture descriptors are still required as several papers [5]1011 rely on texture features derived from grey-level cooccurrence matrices (GLCM) 12 , oriented filters from Gaussian derivatives 7 or local binary patterns (LBP) [8]. The performance of these methods depends on the arbitrary choice of scales and/or orientations as well as a necessary grey-level reduction for GLCMs, the latter entailing the risk of loosing precious information contained in the full bit depth of the original image. Wavelets and filtering techniques have the advantage of providing continuous responses when compared to the binary or categorical outputs of GLCMs or LBPs, which allows for a finer detection and quantification of transients in medical images and were successfully used for lung texture classification in [71314]. Specific wavelet transforms yield multiscale, multi-orientation with infinitesimal angular precision (i.e., steerable filterbanks) and translation invariant (i.e., undecimated transforms) analysis, which allows to characterize textures without making a priori choices on the affine parameters 15 .

In previous work 139] we used isotropic wavelet frames enabling texture analysis with translation and scale covariance as well as rotation invariance. The use of isotropic analysis was based on the assumption that no prevailing orientations are contained in the lung tissue patterns of 2D axial slices in HRCT. Three research contributions are proposed in this article. First, a novel texture characterization approach based on the Riesz transform yielding translation, scale and rotation covariance is introduced. Second, the assumption that lung tissue patterns are locally rotation-invariant is investigated by aligning textures using the local prevailing orientation. Third, principal component analysis (PCA) of the Riesz features is used to obtain templates that are discriminative for lung textures. The approaches are evaluated and compared using a dataset of 85 
ILD cases with a large variety of lung tissue types and a realistic validation scheme based on a leave-one-patient-out (LOPO) cross-validation (CV). We used 13808 overlapping blocks from 2037 manually drawn regions of interest in 1225 2D HRCT slices to validate the proposed methods. A quantitative performance comparison with optimized GLCMs is carried out.

\section{Material and Methods}

\subsection{Dataset}

A database of 85 ILD cases containing HRCT image series with a slice thickness of $1 \mathrm{~mm}$, inter-slice distance of $10 \mathrm{~mm}$ and hand-drawn regions annotated in a collaborative fashion by two radiologists with 15 and 20 years of experience at the University Hospitals of Geneva (HUG) [16] is used to evaluate the performance of the proposed approaches. The diagnosis of each case was confirmed either by pathology (biopsy, bronchoalveolar washing) or by a laboratory/specific test. Based on [4], the texture classes are defined as healthy and four pathological lung tissue types (i.e., ground glass, fibrosis, micronodules and emphysema) that are used to characterize the most frequent ILDs in HRCT. The distribution of the annotated regions and patients is detailed in Table 1.

\subsection{Texture Analysis with Nth-Order Riesz Transforms}

The Riesz transform is a multidimensional extension of the Hilbert transform, which maps any function $f(x)$ to its harmonic conjugate and is a very powerful tool for mathematical manipulations of periodic signals [17. For a twodimensional signal $f(\boldsymbol{x})$, the different components of the $N$ th-order Riesz transform $\mathcal{R}$ are defined in the Fourier domain as

$$
\widehat{\mathcal{R}^{\left(n_{1}, n_{2}\right)}} f(\boldsymbol{\omega})=\sqrt{\frac{n_{1}+n_{2}}{n_{1} ! n_{2} !}} \frac{\left(-j \omega_{1}\right)^{n_{1}}\left(-j \omega_{2}\right)^{n_{2}}}{\|\boldsymbol{\omega}\|^{n_{1}+n_{2}}} \hat{f}(\boldsymbol{\omega}),
$$

for all combinations of $\left(n_{1}, n_{2}\right)$ with $n_{1}+n_{2}=N$ and $n_{1,2} \in \mathbb{N} . \hat{f}(\boldsymbol{\omega})$ denotes the Fourier transform of $f(\boldsymbol{x})$, where the vector $\boldsymbol{\omega}$ is composed by $\omega_{1,2}$ corresponding to the frequencies in the two image axes. The multiplication by $j \omega_{1,2}$ in the numerator corresponds to partial derivatives of $f$ and the division by the norm of $\boldsymbol{\omega}$ in the denominator results in only phase information being retained. Therefore, the 1st-order $\mathcal{R}$ corresponds to an allpass filterbank with directional (singular) kernels $h_{1,2}$ :

$$
\boldsymbol{R} f(\boldsymbol{x})=\left(\begin{array}{l}
\mathcal{R}^{1,0} \\
\mathcal{R}^{0,1}
\end{array}\right)=\left(\begin{array}{l}
h_{1}(\boldsymbol{x}) * f(\boldsymbol{x}) \\
h_{2}(\boldsymbol{x}) * f(\boldsymbol{x})
\end{array}\right),
$$

where

$$
h_{1,2}(\boldsymbol{x})=\frac{x_{1,2}}{2 \pi\|\boldsymbol{x}\|^{3}},
$$

and $x_{1,2}$ correspond to the axes of the image [15]. The Riesz transform commutes with translation, scaling or rotation. The orientation of the Riesz components 


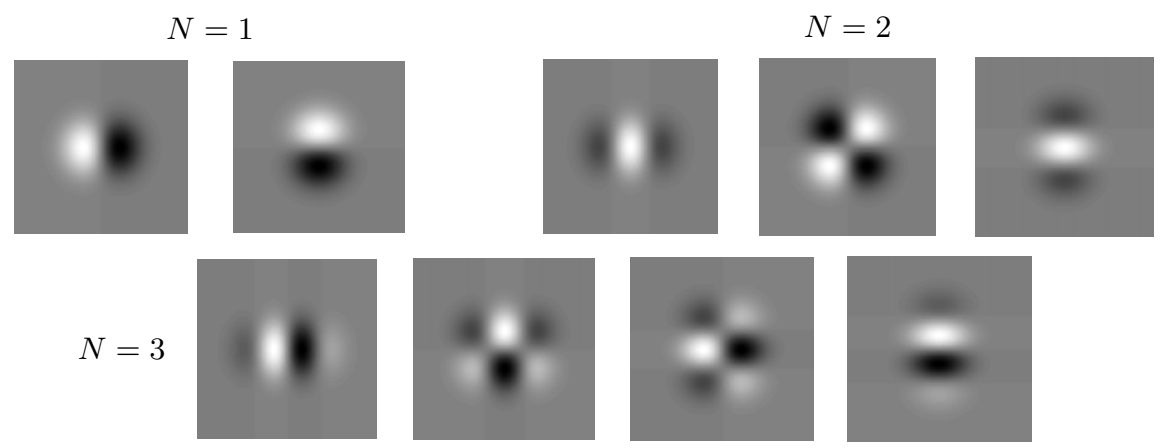

Fig. 1. Templates corresponding to the Riesz kernels convolved with a Gaussian smoother for $N=1,2,3$

is determined by the partial derivatives in Eq. (1). Whereas $2^{N}$ Riesz filters are generated by (10), only $N+1$ components have distinct properties due to commutativity of the convolution operators in (2) (e.g., $\partial^{2} / \partial x \partial y$ is equivalent to $\left.\partial^{2} / \partial y \partial x\right)$. The Riesz components yield a steerable filterbank [15] allowing to analyze textures in any direction, which is an advantage when compared to classical Gaussian derivatives or Gabor filters. Qualitatively, the first Riesz component of even order corresponds to a ridge profile whereas for odd ones we obtain an edge profile, but much richer profiles can be obtained by linear combinations of the different components. The templates of $h_{1,2}(\boldsymbol{x})$ convolved with Gaussian kernels for $N=1,2,3$ are depicted in Fig. 1. The $N$ th-order Riesz transform can be coupled with an isotropic multiresolution decomposition (e.g., Laplacian of Gaussian (LoG)) to obtain rotation-covariant (steerable) basis functions [15].

The main idea of the proposed approach is to derive texture signatures from multiscale Riesz coefficients. An example showing healthy and fibrosis tissue represented in terms of their Riesz components with $N=2$ is depicted in Fig. 2]a). In order to provide a local categorization of the lung parenchyma, lung regions in $2 \mathrm{D}$ axial slices are divided into $32 \times 32$ overlapping blocks with a distance between contiguous block centers of 16 . The Riesz transform is applied to each block, and every Riesz component $n=1, \ldots, N+1$ is mapped to a multiscale representation by convolving them with four LoG filters of scales $s=1, \ldots, 4$ with a dyadic scale progression. In a total of $(N+1) \times 4$ subbands, the variances $\sigma_{n, s}$ of the coefficients are used as texture features along with 22 grey level histogram (GLH) bins in [-1050;600] Hounsfield Units (HU). The percentage of air pixels with values $\leq-1000 \mathrm{HU}$ completes the feature space learned by support vector machines (SVM) with a Gaussian kernel.

The local dominant texture orientations have an influence on the repartition of respective responses of the Riesz components, which is not desirable for creating robust features with well-defined clusters of instances. For example, a rotation of $\pi / 2$ will switch the responses of $h_{1}$ and $h_{2}$ for $N=1$. To ensure that the repartitions of $\sigma_{n, s}$ are comparable for two similar textures having distinct local 


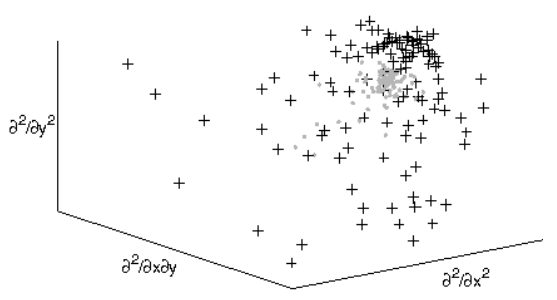

a)

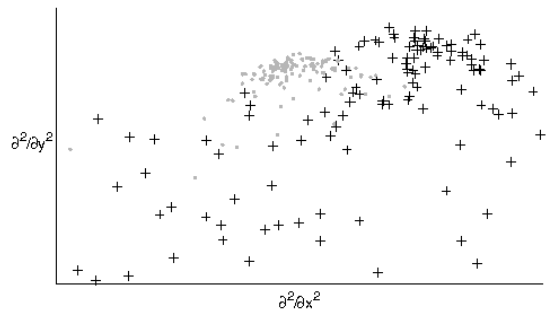

b)

Fig. 2. Riesz representation of healthy (gray dots) versus fibrosis (black crosses) patterns. a) Initial Riesz coefficients in 3D. b) The Riesz coefficients in 2D after having locally aligned the texture based on local prevailing orientation. The component corresponding to $\partial^{2} / \partial x \partial y$ is zero after local rotation and is not shown in $\mathrm{b}$ ).

prevailing orientations, the filters are oriented to have maximal response along $h_{1}$. The dominant orientation $\theta_{d o m}$ of $h_{1}$ at the position $\boldsymbol{x}_{p}$ is

$$
\theta_{d o m}\left(\boldsymbol{x}_{p}\right)=\underset{\theta \in[0, \pi]}{\arg \max }\left(\left(h_{1}^{(\theta)} * g\right) * f\right)\left(\boldsymbol{x}_{p}\right),
$$

where $h_{1}^{(\theta)}(\boldsymbol{x})$ is $h_{1}$ rotated by $\theta$ and $g(\boldsymbol{x})$ is a Gaussian kernel. A local orientation is obtained by rotating every Riesz filter $h_{n}$ with $\theta_{d o m}$ and is done analytically [15]. 2nd--order Riesz coefficients of healthy and fibrosis tissue after local orientation are shown in Fig. 2 b).

\section{Results}

The proposed methods are evaluated using blocks from annotated ROIs with a LOPO CV of 85 patients. For each fold, the cost $C$ of the SVMs and the width $\sigma_{k}$ of the Gaussian kernel are optimized with the training set where parameters allowing best classification accuracy on the training set are found with a grid search $\left(C \in[0.1,1000], \sigma_{k} \in\left[10^{-2}, 10^{1}\right]\right)$ and a 5 -fold $\mathrm{CV}$. The classification performances are compared with optimized GLCMs that are extensively used for lung texture analysis in the literature. Texture features derived from GLCMs are contrast, correlation, energy and homogeneity for various pixel distances $d=1, \ldots, 5$ and orientations $\theta=0, \pi / 4, \pi / 2,3 \pi / 4$, similarly to 8 . Three greylevel reductions are compared: 8, 16 and 32 levels $l$. Optimized SVMs learn in the feature space spanned by concatenated GLCM attributes from every spacing and orientation parameters as well as GLH and air percentage. Classification accuracies using Riesz features of various orders $(N=1, \ldots, 13)$ are compared before and after local rotation in Fig. 3 a). A class-specific performance comparison of best setups for Riesz, Riesz with local orientation, and GLCMs is shown in Fig. $3 \mathrm{~b}$ ). The confusion matrix of the best performing technique ( $N=6$ with local orientation) is detailed in Table 1. In Fig. 4, the distributions of the classes in 


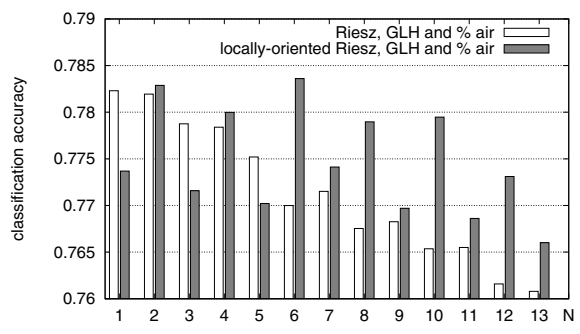

a)

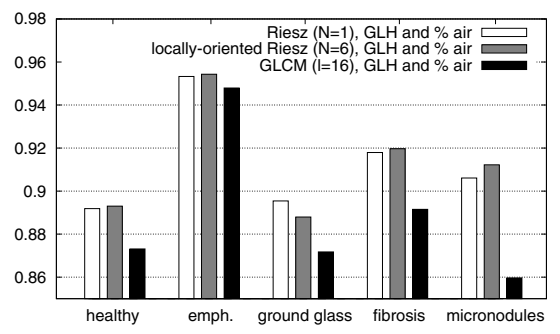

b)

Fig. 3. a) Global classification accuracies with $N=1, \ldots, 13 . N=6$ with local orientation reaches best performance with $78.3 \%$ correct predictions of 13808 instances. b) Class-specific accuracies of the two best configurations $(N=1$ and $N=6$ with orientation) and best performance of GLCMs $(l=16)$.

Table 1. Confusion matrix in $\%$ of the best performing setup ( $N=6$ with local orientation). The numbers of blocks and patients used for the evaluation are detailed. Note that a patient may have several types of lung tissue disorders.

\begin{tabular}{lccccc|cc}
\hline & healthy & emphysema & ground glass & fibrosis & micronodules & \# blocks & \# patients \\
\hline healthy & $\mathbf{7 7 . 5}$ & 7.6 & 4.1 & 0 & 10.7 & 1975 & 7 \\
emphysema & 8.4 & $\mathbf{7 3 . 3}$ & 5.9 & 6.2 & 6.2 & 1298 & 6 \\
ground glass & 14.1 & 0.5 & $\mathbf{7 2 . 3}$ & 10 & 3.1 & 3513 & 32 \\
fibrosis & 0.7 & 2.6 & 8.4 & $\mathbf{8 4 . 5}$ & 3.8 & 3554 & 37 \\
micronodules & 11.6 & 0.7 & 3.5 & 3.7 & $\mathbf{8 0 . 5}$ & 3468 & 16 \\
\hline
\end{tabular}

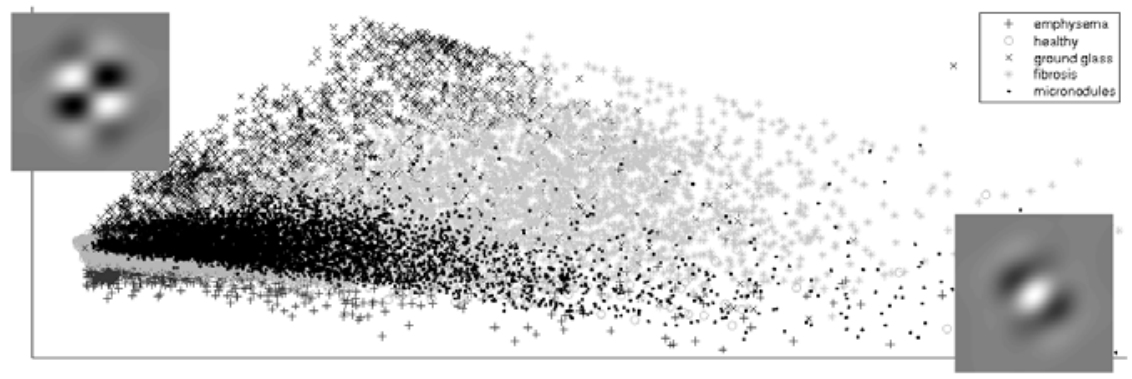

Fig. 4. Visualization of the feature space projected on the two dominant principal components. The corresponding Riesz templates (scale 2) are shown on the axes.

terms of the two dominant principal components of the 6th-order Riesz features with local orientation are shown. The coefficients from the PCA components are used to weight each Riesz component and create learned templates represented on axes in Fig 4. 


\section{Discussions and Conclusions}

We propose a novel texture classification method based on the Riesz transform to categorize lung tissue patterns in HRCT image series from patients affected with ILDs. Compared to the literature, the Riesz features allow to analyze lung texture without prior assumptions on the prevailing scales and orientations and show higher classification performance than popular GLCM texture features. The inherent local orientations of the lung texture are studied by locally steering the Riesz components before further classification. An optimal order of $N=6$ with local orientation allows best classification performance with $78.3 \%$ correct predictions. Fig. 3 a) shows that even orders of the Riesz transform are providing best results, which suggests that ridge detectors are more appropriate than edge detectors for lung texture characterization. Fig. $3 \mathrm{~b}$ ) shows that all lung tissue types except ground glass are better classified with local orientation. All lung tissue types benefit from Riesz texture features when compared to GLCM features. Classes with highest improvement are healthy, fibrosis and micronodules, which are those containing most texture information. The performance comparisons are statistically significant for all classes $(p \leq 0.0002)$ but emphysema $(p=0.073)$. An absolute gain of $6.1 \%$ in global classification accuracy is obtained with Riesz and rotation adjustment (78.3\%) when compared to GLCM $(72.2 \%)$. This suggests that the arbitrary choices of scale and orientation parameters are not optimal for accurate characterization of the lung texture, although these values are the most commonly used in the literature 56710118. Table 1 shows that healthy and ground glass patterns are the most challenging to separate due to high intra-class variability among patients and severity of disease . Confusion between micronodules and healthy tissue is observed, which is a limitation of $2 \mathrm{D}$ approaches as bronchovascular structures have similar appearance as micronodules in the 2D axial slices. Unfortunately, the HRCT imaging protocol is very anisotropic with a gap between slices of $10 \mathrm{~mm}$ and does not allow for full 3D texture analysis. The Riesz features are easily extendable to three dimensions [15] to reduce the confusions between micronodules and healthy bronchovascular structures in isotropic multidetector CT. The balanced performance achieved among the various classes of lung tissue suggest that the proposed features are efficient to analyze lung tissue patterns for a large variety of ILD diagnoses. The ability of the Riesz features to adapt to lung textures is illustrated in Fig. 4 where templates according to the dominant principal components of the feature space are shown. In future work, feature selection and learning methods will be incorporated to promote the most relevant Riesz components and reduce the influence of noise. Thanks to the affine-covariant properties of the proposed methods, they are expected to provide tools for analyzing textures with no prior assumptions on translation, scale and orientation parameters in various applications.

Acknowledgments. This work was supported by the Swiss National Science Foundation (under grants 205321-130046 and PP00P2-123438), the CIBM, and the EU 7th Framework Program in the context of Khresmoi (FP7-257528). 


\section{References}

1. Tourassi, G.D.: Journey toward computer-aided diagnosis: Role of image texture analysis. Radiology 213(2), 317-320 (1999)

2. Berlin, L.: Malpractice issues in radiology. perceptual errors. AJR Am. J. Roentgenol. 167(3), 587-590 (1996)

3. Castellano, G., Bonilha, L., Li, L.M., Cendes, F.: Texture analysis of medical images. Clin. Radiol. 59(12), 1061-1069 (2004)

4. Webb, W.R., Müller, N.L., Naidich, D.P.: High-Resolution CT of the Lung. Lippincott Williams \& Wilkins, Philadelphia (2001)

5. Delorme, S., Keller-Reichenbecher, M.A., Zuna, I., Schlegel, W., Van Kaick, G.: Usual interstitial pneumonia: Quantitative assessment of high-resolution computed tomography findings by computer-assisted texture-based image analysis. Invest. Radiol. 32(9), 566-574 (1997)

6. Uppaluri, R., Hoffman, E.A., Sonka, M., Hunninghake, G.W., McLennan, G.: Interstitial lung disease: A quantitative study using the adaptive multiple feature method. Am. J. Respir. Crit. Care Med. 159(2), 519-525 (1999)

7. Sluimer, I.C., van Waes, P.F., Viergever, M.A., van Ginneken, B.: Computer-aided diagnosis in high resolution CT of the lungs. Med. Phy. 30(12), 3081-3090 (2003)

8. Sørensen, L., Shaker, S.B., de Bruijne, M.: Quantitative analysis of pulmonary emphysema using local binary patterns. IEEE Trans. Med. Imaging 29(2), 559$569(2010)$

9. Depeursinge, A.: Affine-invariant texture analysis and retrieval of 3D medical images with clinical context integration. PhD thesis, University of Geneva (2010)

10. Xu, Y., Sonka, M., McLennan, G., Guo, J., Hoffman, E.A.: MDCT-based 3D texture classification of emphysema and early smoking related lung pathologies. IEEE Trans. Med. Imaging 25(4), 464-475 (2006)

11. Caban, J.J., Yao, J., Avila, N.A., Fontana, J.R., Manganiello, V.C.: Texture-based computer-aided diagnosis system for lung fibrosis. In: Giger, M.L., Karssemeijer, N. (eds.) Medical Imaging 2007: Computer-Aided Diagnosis, vol. 6514. SPIE, San Jose (2007)

12. Haralick, R.M., Shanmugam, K., Dinstein, I.: Textural features for image classification. IEEE Trans. Syst. Man and Cybern. 3(6), 610-621 (1973)

13. Depeursinge, A., Sage, D., Hidki, A., Platon, A., Poletti, P.A., Unser, M., Müller, H.: Lung tissue classification using Wavelet frames. In: Conf. Proc. IEEE Eng. Med. Biol. Soc., pp. 6259-6262. IEEE Computer Society, Los Alamitos (2007)

14. Sørensen, L., Lo, P., Ashraf, H., Sporring, J., Nielsen, M., de Bruijne, M.: Learning COPD sensitive filters in pulmonary CT. In: Yang, G.-Z., Hawkes, D., Rueckert, D., Noble, A., Taylor, C. (eds.) MICCAI 2009. LNCS, vol. 5762, pp. 699-706. Springer, Heidelberg (2009)

15. Unser, M., Van De Ville, D.: Wavelet steerability and the higher-order Riesz transform. IEEE Trans. Imag. Proc. 19(3), 636-652 (2010)

16. Depeursinge, A., Vargas, A., Platon, A., Geissbuhler, A., Poletti, P.A., Müller, H.: Building a reference multimedia database for interstitial lung diseases. Comput. Med. Imaging. Graph. (to appear)

17. Stein, E.M., Weiss, G.: Introduction to Fourier analysis on Euclidean spaces. Princeton University Press, Princeton (1971) 